\title{
Reducing star leakage with a nuller coronagraph
}

\section{An analytic approach for a Sonine apodized circular aperture}

\author{
C. Aime ${ }^{1}$, Y. Rabbia ${ }^{1}$, A. Carlotti ${ }^{1,2}$, and G. Ricort ${ }^{1}$ \\ ${ }^{1}$ Université de Nice Sophia-Antipolis, Centre National de la Recherche Scientifique, Laboratoire Fizeau, \\ Observatoire de la Côte d'Azur, Parc Valrose, 06108 Nice, France \\ e-mail: [claude.aime;gilbert.ricort]@unice.fr; yves.rabbia@obs-azur.fr; acarlott@princeton.edu \\ ${ }^{2}$ Princeton University, Mechanical \& Aerospace Engineering, Olden street, Princeton, 08544 NJ, USA
}

Received 21 December 2010 / Accepted 29 March 2011

\section{ABSTRACT}

\begin{abstract}
Aims. Our objective is to test the improvement of the detection capabilities of a nuller coronagraph when it is coupled to an apodizer. We call this instrument an apodized nuller coronagraph (ANC).

Methods. With the usual Fourier optics formalism we first derive the analytical expression of the intensity distribution for an off-axis point-like source in the aperture and the focal plane of an ANC. Then the star leakage is obtained by integration of this response over the angular extension of the star. In this process are included the star's angular diameter, the apodization strength and the diameter of the telescope. Eventually we consider an application to the specific case of Sonine apodization.

Results. From this analytical approach we obtain expressions of the contrast and signal-to-noise ratio for the direct detection of an exoplanet. These expressions involving instrumental parameters allow one to optimize the required minimum telescope's diameter and apodization strength with respect to the detection of a given target in spite of star leakage. We show that the direct detection of an exoplanet in a solar-like system can be obtained with a nuller coronagraph as soon as the aperture is sufficiently apodized, although the energy throughput of the system is reduced. For a system at $10 \mathrm{pc}$, an 8-m class telescope appears necessary to detect an exo-Earth, while no larger than a 2-m class telescope is required for an exo-Jupiter.
\end{abstract}

Key words. instrumentation: high angular resolution - instrumentation: interferometers - techniques: high angular resolution techniques: interferometric - methods: analytical

\section{Introduction}

By the end of 2010 the community knew about more than five hundred exoplanets. An interactive catalog of the discoveries of exoplanets, categorized by detection type, can be found in The Extrasolar Planets Encyclopaedia at exoplanet . eu, an URL remarkably held up to date since 1995. Another catalog can be found at exoplanets . org (Wright et al. 2011). A large majority of these planets has been detected with the radial velocity method. The transit technique (Seager \& Sasselov 2000) was used for example with the Corot and Kepler satellites, which provide semi-direct detections, but the ultimate goal is to collect photons from Earth-like exoplanets for a direct spectral analysis.

So far images of 12 planetary systems have been obtained with coronagraphic instruments, adaptive optics, and data processing techniques. The targeted stars are young and they have mostly been observed in the infrared, allowing the discovery of young, therefore warm and bright, companions. Ideally, an imaging instrument should be able to detect older, therefore colder and fainter, planets, such as those of our own 4.6 billion years old solar system, which are expected to be dramatically fainter than their parent star, typically at the level of $10^{-6}$ in the thermal infrared and down to $10^{-10}$ in the visible.

The last fifteen years have seen considerable efforts to devise concepts and instruments able to perform these observations. There are several ways to classify them (Perryman 2000; Aime 2007; Guyon 2009; Oppenheimer \& Hinkley 2009).
These concepts include external occulters (Cash 2006; Vanderbei et al. 2007; Arenberg et al. 2007), nuller interferometers (Bracewell 1978), various pupil apodization techniques (Nisenson \& Papaliolios 2001; Kasdin et al. 2003; Guyon 2003; Aime 2005), and coronagraphs (Gay \& Rabbia 1996; Roddier \& Roddier 1997; Rouan et al. 2000; Mawet et al. 2005; Guyon et al. 2006).

These instrumental techniques work each in a different way, but they share a common objective, which is to gather as many planet photons as possible in the broadest bandwidth and in the shortest exposure time. Thus they have to suppress the diffracted starlight as much as possible over a broad enough spectral bandwidth and with the highest possible throughput, which is beneficial for the detection performance. Moreover, they should have a small inner working angle (IWA) for angular resolution capabilities, though this feature makes them sensitive to star leakage for a star with a finite diameter. Removing this kind of drawback is the role of the coupling to an apodizer.

In this paper we focus on nuller coronagraphs, a type of coronagraphs whose first model was the achromatic interferocoronagraph (AIC, Gay \& Rabbia 1996). The AIC is a system that is at the same time a coronagraph and a nulling interferometer. The technique has been described in several papers (Rabbia et al. 1998; Baudoz et al. 2000a,b) and is recalled in Sect. 2. Although AIC is based on a Michelson interferometer, some other nuller coronagraphs are based on different interferometers: the Sagnac interferometer (Tavrov et al. 2005) and the MachZehnder interferometer (Allouche et al. 2009). 
Nuller coronagraphs have the ability to fully reject the light of an on-axis point-like source. They possess a very narrow inner working angle (IWA) with the drawback that their efficiency degrades when the coherence of the wavefront is reduced because of the angular diameter of the source. To rule out this problem, Tavrov et al. (2008) proposed to use a double pass, and we have recently proposed a variant of the AIC called the achromatic rotation-shearing coronagraph (ARC) in Aime et al. (2010), cited as Paper I here.

In the present paper we develop the idea suggested in Paper I to couple an apodizer and a nuller coronagraph to reduce the star leakage. In this regard our approach is similar to the numerical study described in Nishikawa et al. (2005) for a somewhat different device. As already noted by these authors, apodization and nulling coronagraphy have complementary effects. The nuller coronagraph provides a strong rejection for an on-axis point source, while apodization makes the background (diffraction wings) at the location of the planet image faint enough to render it detectable, with the drawbacks of an increased IWA and a decrease of throughput. Though we consider the coupling of an apodizer with AIC, this coupling is immediately applicable to another nuller coronagraph.

The apodization we consider here consists in giving a smooth transmission with a circular geometry to the exit pupil. We use Sonine functions as transmission profile, and the main interest of these functions is that they allow us to derive analytically the theoretical performance of the system. We can thus stress the dramatic influence of star leakage on the detectability of the planet, and then show how apodization can greatly improve this capability. Optimizing the transmission, contrast, and IWA associated with the apodization profile is a different problem, and it will be but briefly considered at the end of the paper.

The paper is organized as follows. Section 2 gives the general expressions for an apodized nuller coronagraph. Section 3 consider the case when a Sonine apodization is used. Section 4 provides simple analytical expressions for the contrast and for the signal-to-noise ratio $(S N R)$. Section 5 presents a discussion and gives a conclusion.

\section{General expressions for an apodized nuller coronagraph (ANC)}

In this section we focus on the AIC, which is the concept behind the whole class of nuller coronagraphs (NC). Because it has been described extensively (see the papers mentioned above, starting with Gay \& Rabbia 1996) only short reminders are given here for the reader's convenience.

The AIC is basically a Michelson Fourier interferometer (and as such it has two output beams), modified by inserting a subsystem (cat's eye) along one arm providing both an achromatic $\pi$ phase shift and a 180 degrees pupil rotation (around the propagation axis). A focusing component is used to form the images in the focal plane. For an on-axis point source, the recombination of the waves results in a fully destructive interference at the destructive port, the light being rejected through the constructive port. Alternatively one may consider that in the focal planes two point spread functions (PSF) obtained through the two arms of the instrument are added destructively, thanks to the $\pi$ phase shift. For an off-axis source the system yields two separated images because of the tilted incident wavefront, displayed symmetrically with respect to the axis. No strongly destructive interference occurs if the star-planet separation is larger than a fraction of an Airy radius, and a planet is seen as twin images.
In between these two cases, for a source just slightly off-axis, for example owing to guiding errors, the AIC produces a more complex illumination of the focal plane that is the result of the interference of two complex amplitudes of opposite signs.

If we consider a star perfectly on-axis, but with a finite diameter, the light from the central point of the star will be fully cancelled by the AIC, but not the light coming from the rest of the star. This will result in an unwanted illumination of the focal plane that is called star leakage. The object of this paper is to quantify the star leakage reduction when the Airy images are replaced by aperture responses with lower diffraction wings, which is the effect produced by aperture apodization.

We shall consider below how apodization modifies the overall transmission of the nuller coronagraph for an off-axis point source, and then derive the expression of the star leakage. We restrict ourselves to a circular unobstructed aperture of diameter $D$, whose transmission is modified in amplitude by a function $t(r)$, and the effective pupil transmission is

$P(r)=t(r) \Pi\left(\frac{r}{D}\right)$,

where $r$ is the distance to the center, and $\Pi(r / D)$ equals 1 if $r<$ $D / 2$, and 0 otherwise. This will reduce the effective collecting area to the quantity:

$\Phi=2 \pi \int_{0}^{D / 2} r|t(r)|^{2} \mathrm{~d} r$.

The calculations below draw heavily on Paper I. Let us consider the propagation of a tilted plane wavefront through the telescope and the AIC. On the telescope aperture, the phase term associated with a wavefront coming from a point source in the direction $\alpha_{0}=\rho_{0} \cos \left(\varphi_{0}\right)$ and $\beta_{0}=\rho_{0} \sin \left(\varphi_{0}\right)$ on the sky can be written in cartesian and polar coordinates as

$\exp \left(-\frac{2 \mathrm{i} \pi}{\lambda}\left(\alpha_{0} x+\beta_{0} y\right)\right)=\exp \left(-\frac{2 \mathrm{i} \pi}{\lambda} \rho_{0} r \cos \left(\theta-\varphi_{0}\right)\right)$

where we use $(r, \theta)$ for the polar coordinates in the aperture, with $x=r \cos (\theta)$ and $y=r \sin (\theta)$. The phasors in the above equation are the expressions of the incoming amplitude for a tilted wavefront in the two systems of coordinates.

As already indicated, a nuller coronagraph such as the AIC works by making the subtraction of two wavefronts rotated one relative to the other by an angle of $\pi$. It is convenient to write this rotation symmetrically as $\pm \pi / 2$. Changing $\cos \left(\theta-\varphi_{0}\right)$ in Eq. (3) for $\cos \left(\theta-\varphi_{0} \pm \pi / 2\right)= \pm \sin \left(\theta-\varphi_{0}\right)$, the complex amplitude in the subtractive output of the AIC for an incident wave of unit amplitude can be written as

$$
\begin{aligned}
& A\left(r, \theta, \rho_{0}, \varphi_{0}\right)= \\
& \quad \frac{1}{2} P(r)\left[\exp \left(\frac{2 \mathrm{i} \pi}{\lambda} \rho_{0} r \sin \left(\theta-\varphi_{0}\right)\right)-\exp \left(-\frac{2 \mathrm{i} \pi}{\lambda} \rho_{0} r \sin \left(\theta-\varphi_{0}\right)\right)\right] \\
& \quad=\mathrm{i} P(r) \sin \left(\frac{2 \pi}{\lambda} \rho_{0} r \sin \left(\theta-\varphi_{0}\right)\right) .
\end{aligned}
$$

The corresponding intensity is written

$I\left(r, \theta, \rho_{0}, \varphi_{0}\right)=\frac{1}{2}|P(r)|^{2}\left[1-\cos \left(\frac{4 \pi}{\lambda} \rho_{0} r \sin \left(\theta-\phi_{0}\right)\right)\right]$.

The integrated intensity transmitted by the apodized nuller coronagraph for a point source at the angular distance $\rho_{0}$, normalized 
by the flux collected through the apodized aperture, can be written as

$$
\begin{aligned}
\tau\left(\rho_{0}\right) & =\frac{1}{2}-\frac{1}{2 \Phi} \iint|P(r)|^{2} \cos \left(\frac{4 \pi}{\lambda} \rho_{0} r \sin \left(\theta-\phi_{0}\right)\right) r \mathrm{~d} r \mathrm{~d} \theta \\
& =\frac{1}{2}\left(1-\frac{I_{1}\left(\rho_{0}\right)}{\Phi}\right), \text { where } \\
I_{1}\left(\rho_{0}\right) & =2 \pi \int_{0}^{D / 2} r|t(r)|^{2} J_{0}\left(\frac{2 \pi}{\lambda} 2 \rho_{0} r\right) \mathrm{d} r .
\end{aligned}
$$

This latter integral is the Hankel transform of the transmission in intensity (and not in amplitude) of the aperture for the variable $2 \rho_{0} / \lambda$ (and not simply for $\rho_{0} / \lambda$ ). This expression shows that the light transmission at the output of the apodized nuller coronagraph goes from 0 for an on-axis point source $\left(\rho_{0}=0\right.$, and the starlight is perfectly cancelled) up to transmission values that oscillate around 0.5 when $\rho_{0}$ becomes larger than the width of $I_{1}\left(\rho_{0}\right)$.

It is interesting to note that because of the factor 2 in the variable $2 \rho_{0} / \lambda$, the IWA of nuller coronagraphs are twice as small as the telescope nominal resolution. The use of apodization will therefore increase this IWA by a factor governed by the shape of the Hankel transform of the transmission in intensity $|t(r)|^{2}$, as already indicated.

Because the leakage depends on the behavior of $I_{1}\left(\rho_{0}\right)$ for low values of $\rho_{0}$, if the goal is to reduce the effect of an offset caused by a poor centering of the star, one would like to use an apodization function with a flat response near the origin. Unfortunately, because the relevant function is $|t(r)|^{2}$, the on-sky transmission near the origin must follow a $\rho_{0}^{2}$ behavior, no matter what apodization profile is used, and switching between different apodization profiles will only change the coefficient of this quadratic behavior. Examples are given below.

A function that is more relevant than the integrated leakage is the level of the leakage at the planet position. For that we need to express the residual intensity in the focal plane for an off-axis point source. Returning to Eq. (4), we have

$A\left(r, \theta, \rho_{0}, \varphi_{0}\right) \simeq \mathrm{i} P(r) \frac{2 \pi}{\lambda} \rho_{0} r \sin \left(\theta-\varphi_{0}\right)$,

where we made the approximation $\sin (u) \simeq u$. This approximation is legitimate because $\rho_{0}$ is very small, its maximal value being the apparent angular radius of the star.

The scaled Fourier transform in polar coordinates of $A(r, \theta)$ yields the amplitude in the image plane (see Eq. (11) of Paper I) as the function $\hat{A}(\rho, \varphi)$. The derivation is similar to the one given in Appendix A of Paper I, but for the insertion of the variable transmission factor $t(r)$ in the circular aperture of diameter $D$. We have

$\hat{A}(\rho, \varphi)=\mathrm{i} \frac{2 \pi}{\lambda^{2}} \rho_{0} \int_{0}^{D / 2} r^{2} t(r) \int_{0}^{2 \pi} \sin \left(\theta-\varphi_{0}\right) \mathrm{e}^{-2 \mathrm{i} \pi \rho r \cos (\theta-\varphi) / \lambda} \mathrm{d} \theta \mathrm{d} r$.

Here as in Paper I we make $\theta-\varphi=\theta^{\prime}$, and develop $\sin \left(\theta^{\prime}+\right.$ $\left.\varphi-\varphi_{0}\right)=\sin \left(\theta^{\prime}\right) \cos \left(\varphi-\varphi_{0}\right)+\cos \left(\theta^{\prime}\right) \sin \left(\varphi-\varphi_{0}\right)$. The integral on $\sin \left(\theta^{\prime}\right)$ is canceled because of the parity properties of this function. We have

$$
\begin{aligned}
\hat{A}(\rho, \varphi) & =\frac{4 \pi^{2}}{\lambda^{2}} \rho_{0} \sin \left(\varphi-\varphi_{0}\right) \int_{0}^{D / 2} r^{2} t(r) J_{1}\left(\frac{2 \pi \rho r}{\lambda}\right) \mathrm{d} r \\
& =\frac{2 \pi}{\lambda^{2}} \rho_{0} \sin \left(\varphi-\varphi_{0}\right) I_{2}(\rho), \text { where } \\
I_{2}(\rho) & =2 \pi \int_{0}^{D / 2} r^{2} t(r) J_{1}\left(\frac{2 \pi \rho r}{\lambda}\right) \mathrm{d} r .
\end{aligned}
$$

This latter integral is the first order Hankel transform of the function $r t(r)$ for the variable $\rho / \lambda$, including in our definitions of Hankel transforms the factor $2 \pi$. The intensity becomes

$I(\rho, \varphi)=|\hat{A}(\rho, \varphi)|^{2}=\frac{4 \pi^{2}}{\lambda^{4}} \rho_{0}^{2} \sin ^{2}\left(\varphi-\varphi_{0}\right) I_{2}^{2}(\rho)$.

Because of the term $\sin ^{2}\left(\varphi-\varphi_{0}\right)$, the residual intensity $I(\rho, \phi)$ is not a radial function. Still, considering the approximation where $\rho_{0}$ is small, $I(\rho, \phi)$ increases as $\rho_{0}^{2}$.

The leakage energy distribution is obtained through the integration over the angular brightness distribution of the star (of angular diameter $\Theta$ ):

$$
\begin{aligned}
L(\rho, \varphi) & =\frac{4}{\pi \Theta^{2}} \frac{4 \pi^{2}}{\lambda^{4}} I_{2}^{2}(\rho) \int_{0}^{\Theta / 2} \rho_{0}^{3} \mathrm{~d} \rho_{0} \int_{0}^{2 \pi} \sin ^{2}\left(\varphi-\varphi_{0}\right) \mathrm{d} \varphi_{0} \\
& =L(\rho)=\frac{\pi^{2} \Theta^{2}}{4 \lambda^{4}} I_{2}^{2}(\rho) .
\end{aligned}
$$

The resulting function is now a radial function, and we normalized the expression on the angular surface of the star $\pi \Theta^{2} / 4$, to handle the star brightness and star diameter separately. To obtain an analytical expression for the star leakage, we have to use an aperture transmission $t(r)$ whose integral in the above relation can be written as known functions. This is the case if we use for $t(r)$ the so-called Sonine functions. We briefly recall the properties of these functions in the next section before using them for the ANC.

\section{Expressions for a circular Sonine ANC}

\subsection{Sonine-apodized circular aperture and corresponding PSF}

The transmission in amplitude of a radial Sonine apodization of a circular aperture of diameter $D$ can be written

$t(r)=t_{s}(r)=\left(1-4 \frac{r^{2}}{D^{2}}\right)^{s}$

where $s$ is the so-called Sonine parameter that defines the apodization's strength. The value $s=0$ corresponds to the unapodized case. Illustrations of the apodized transmission $t_{s}(r)$ for a few values of the Sonine parameter $s$ are shown in Fig. 1. The curves are drawn for an aperture of diameter unity.

The point-spread function (PSF) $R_{s}(\rho)$ in the focal plane of this apodized aperture can be written as (Osterberg \& Wilkins 1949; Jacquinot \& Roizen-Dossier 1964)

$R_{s}(\rho)=\frac{1}{\lambda^{2}}\left|\hat{P}\left(\frac{\rho}{\lambda}\right)\right|^{2}=2^{2 s-2} \frac{\pi^{2} D^{4}}{\lambda^{2}}\left(\frac{\lambda}{\pi \rho D}\right)^{2 s+2} J_{s+1}^{2}\left(\frac{\pi \rho D}{\lambda}\right) \Gamma^{2}(s+1)$,

where $\lambda$ is the light wavelength and $\rho$ is the angular radial distance. We assume a unit amplitude for the incoming wave on the aperture. For integer values of $s, \Gamma(s+1)=s$ !, but $s$ may take any positive real value.

From there we can derive three relevant quantities for further use in this work: the effective collection area $\Phi_{s}$ of the apodized aperture (governing the energy throughput), the value of the PSF at the origin $\left(R_{s}(0)\right)$, and the equivalent angular area $\triangle_{s}$ of the 


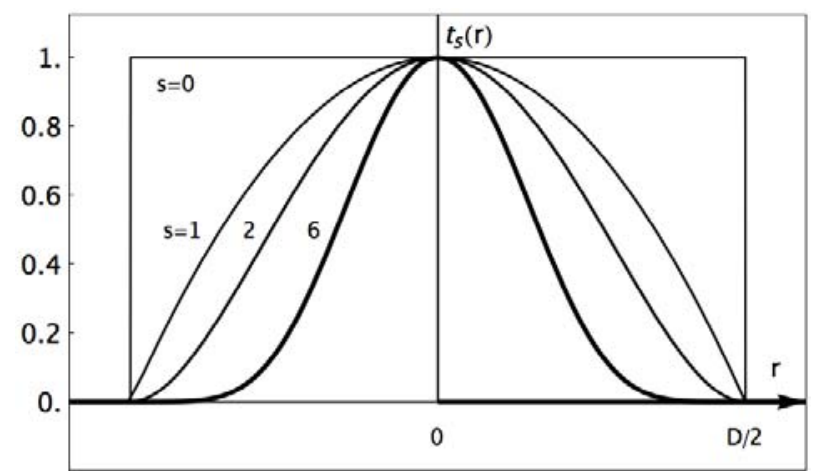

Fig. 1. Radial cuts of the aperture transmission (in amplitude) modified by the Sonine functions $t_{s}(r)$ given in Eq. (12), for $s=0$ (clear aperture), 1,2 , and 6 .

PSF. Following the approach given in Aime (2005), we have

$$
\begin{aligned}
& \Phi_{s}=\int_{0}^{\infty} 2 \pi r P_{s}^{2}(r) \mathrm{d} r=\frac{\Phi_{0}}{1+2 s} \\
& R_{s}(0)=\frac{1}{\lambda^{2}}\left|\int_{0}^{\infty} 2 \pi r P_{s}(r) \mathrm{d} r\right|^{2}=\frac{R_{0}(0)}{(1+s)^{2}} \\
& \triangle_{s}=\frac{1}{R_{s}(0)} \int_{0}^{\infty} 2 \pi r R_{s}(r) \mathrm{d} r=\frac{\Phi_{s}}{R_{s}(0)}=\triangle_{0} \frac{(1+s)^{2}}{1+2 s},
\end{aligned}
$$

where the quantities $\Phi_{0}, R_{0}(0)$ and $\triangle_{0}$ refer to a $\mathrm{NC}$ used with a clear aperture:

$\Phi_{0}=\frac{\pi D^{2}}{4}, R_{0}(0)=\frac{\pi^{2} D^{4}}{16 \lambda^{2}}$, and $\Delta_{0}=\frac{\Phi_{0}}{R_{0}(0)}=\frac{4 \lambda^{2}}{\pi D^{2}}$.

It is interesting to note that the effect of apodization simply is a multiplicative factor.

For high enough values of its argument, the Bessel function can be approximated and majored by

$J_{n}(z) \simeq \sqrt{\frac{2}{\pi z}} \cos (z-(2 n+1) \pi / 4) \leq \sqrt{\frac{2}{\pi z}}$.

This approximation is fair enough even for low values (as soon as $z$ is farther than the second lobe), and the last term of the equation gives the envelope of the function. By substituting this expression for the Bessel function into $R_{s}(\rho)$, we have

$$
\begin{aligned}
& R_{s}(\rho) \simeq \tilde{R}_{s}(\rho)=\tilde{R}_{0}(\rho) \times g_{s}(\rho), \text { with } \\
& \tilde{R}_{0}(\rho)=\frac{\lambda D}{2 \pi^{2} \rho^{3}}, \text { and } \\
& g_{s}(\rho)=4^{s} \Gamma^{2}(s+1)\left(\frac{\lambda}{\pi \rho D}\right)^{2 s} .
\end{aligned}
$$

This approximation shows that the diffraction wings of the Sonine-apodized PSF decrease as $\rho$ to the power $3+2 s$, against just to the power 3 for the Airy pattern. This interesting behavior is obtained, however, at the expense of a reduced transmission and a larger size of the central part of the PSF.

An illustration of $R_{s}(\rho)$ for $s$ equal to 0 (the Airy pattern), 2, and 6 respectively is shown in Fig. 2 together with the approximate expression $\tilde{R}_{s}(\rho)$. This figure clearly shows that $\tilde{R}_{s}(\rho)$ is a very fair estimate of the envelope of $R_{s}(\rho)$, as soon as $\rho$ is larger than the central part of the PSF.

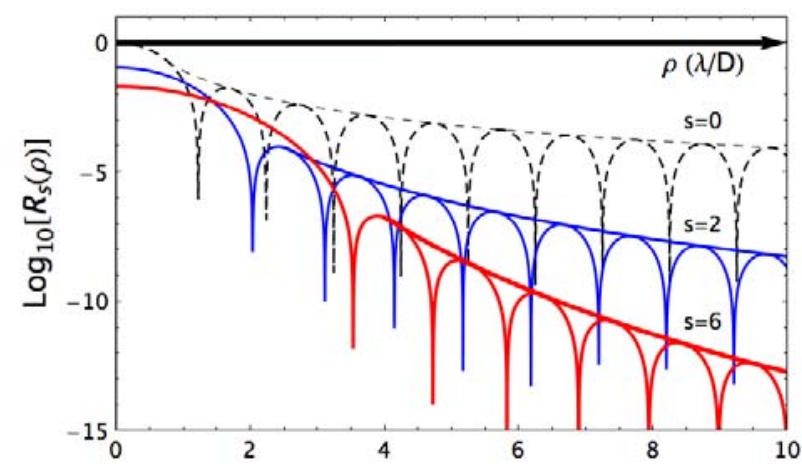

Fig. 2. PSF $R_{s}(\rho)$ and their envelopes in a logarithmic scale, given in Eq. (12), for $s=0$ (dashed line), 2 and 6 (full lines). The angular distance $\rho$ is in units of $\lambda / D$. The envelopes starts at $1,2.5$ and $4 \lambda / D$ respectively.

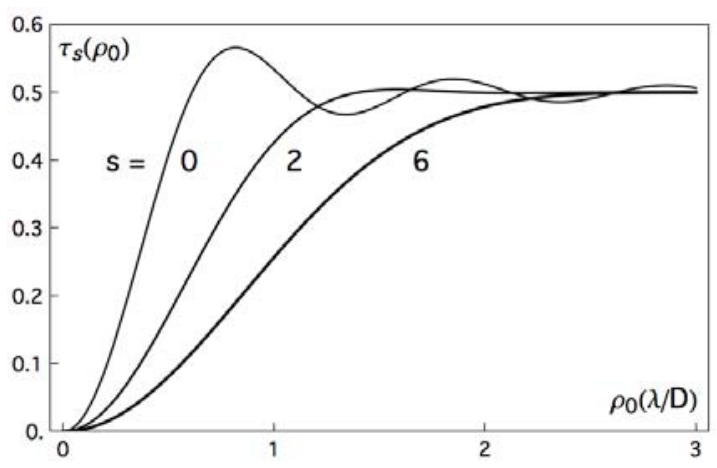

Fig. 3. On-sky normalized transmission $\tau_{s}\left(\rho_{0}\right)$ of an ANC for $s=0$ (no apodization), 2, 6. Angular distance is in units of $\lambda / D$.

\subsection{Star leakage for a Sonine ANC}

When using Sonine apodizations, the integrals $I_{1}\left(\rho_{0}\right)$ and $I_{2}(\rho)$ introduced in Eqs. (6) and (9) become

$$
\begin{aligned}
& I_{1, s}\left(\rho_{0}\right)=\frac{\pi D^{2}}{4}\left(\frac{\lambda}{\pi \rho_{0} D}\right)^{2 s+1} J_{2 s+1}\left(\frac{2 \pi \rho_{0} D}{\lambda}\right) \Gamma(2 s+1) \\
& I_{2, s}(\rho)=\frac{\pi D^{3}}{4} 2^{s}\left(\frac{\lambda}{\pi \rho D}\right)^{s+1} J_{s+2}\left(\frac{\pi \rho D}{\lambda}\right) \Gamma(s+1) .
\end{aligned}
$$

We can rewrite the integrated and normalized intensity transmitted by the ANC for a point source off-axis by $\rho_{0}$ as given in Eq. (6)

$\tau_{s}\left(\rho_{0}\right)=\frac{1}{2}\left[1-\left(\frac{\lambda}{\pi \rho_{0} D}\right)^{2 s+1} J_{2 s+1}\left(\frac{2 \pi \rho_{0} D}{\lambda}\right) \Gamma(2 s+2)\right]$.

In Fig. 3 we display the on-sky transmission profiles $\tau_{s}\left(\rho_{0}\right)$ of the ANC for various values of $s$. These profiles are normalized to 0.5 for each value of $s$. Evidently increasing the apodization strength results in a decrease of the transmission for slightly offaxis point-like sources. They either come from small pointing errors or from the finite diameter of the star. Besides, the apodization globally decreases the overall transmission and it widens the IWA.

Using for $t(r)$ the Sonine transmission $t_{s}(r)$, we have a new expression for the intensity of the residual light from an off-axis point-like source (as given in Eq. (10)):

$I(\rho, \varphi)=$

$4^{s-1} \frac{\pi^{2} D^{4}}{\lambda^{2}} \frac{\rho_{0}^{2}}{\rho^{2}} \sin ^{2}\left(\varphi-\varphi_{0}\right)\left(\frac{\lambda}{\mathrm{D} \pi \rho}\right)^{2 s} J_{s+2}^{2}\left(\frac{\mathrm{D} \pi \rho}{\lambda}\right) \Gamma^{2}(s+1)$. 

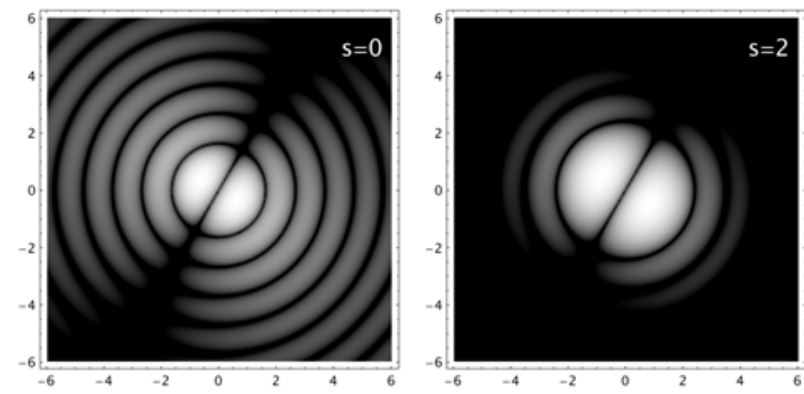

Fig. 4. Illustration of $I(\rho, \varphi)$ for $s=0$ (left) and $s=2$ (right). The intensity (logarithmic scale) was normalized and white corresponds to 1 , while black corresponds to $10^{-3}$. Angular distances are in units of $\lambda / D$.

An illustration of $I(\rho, \varphi)$ for $s=0$ and $s=2$ is given in Fig. 4. In this specific example the off-axis point source's coordinates are $\phi_{0}=\pi / 3$ and $\rho_{0}=0.08 \lambda / D$.

The Sonine transmission of Eq. (12) can also replace $t(r)$ in the expression of the star leakage given in Eq. (11). We then have

$L_{s}(\rho)=4^{s-3} \frac{\pi^{2} D^{4}}{\lambda^{2}} \times \frac{\Theta^{2}}{\rho^{2}}\left(\frac{\lambda}{D \pi \rho}\right)^{2 s} J_{s+2}^{2}\left(\frac{D \pi \rho}{\lambda}\right) \Gamma^{2}(s+1)$.

This circular distribution is the background against which the twin-images of the planet are to be extracted.

Here it is also possible to obtain an approximate expression of the star leakage outside the central region. Replacing again the Bessel function by its approximation, $L_{s}(\rho)$ can be written as the function $\tilde{L}_{s}(\rho)$, envelope of $L_{s}(\rho)$, as follows:

$L_{s}(\rho) \simeq \tilde{L}_{s}(\rho)=\tilde{L}_{0}(\rho) \times g_{s}(\rho)$, with

$\tilde{L}_{0}(\rho)=\frac{D^{3} \Theta^{2}}{32 \lambda \rho^{3}}$

where $\tilde{L}_{0}(\rho)$ is the leakage for the non-apodized AIC as given in Eq. (18) of Paper I, and $g_{s}(\rho)$ is the attenuation factor caused by the apodization, the same already given for the direct PSF response in Eq. (17). This simple result is not surprising since the leakage originates in the finite difference of the two diffraction responses, as already noted in Sect. 2.

It is interesting to estimate the rejection gain obtained using an ANC against a simple apodized aperture. This gain is evaluated by the ratio $\tilde{L}_{s}(\rho)$ to $\tilde{R}_{s}(\rho)$. Making use of the previous expressions, we have

$\frac{\tilde{L}_{s}(\rho)}{\tilde{R}_{s}(\rho)}=\frac{\tilde{L}_{0}(\rho)}{\tilde{R}_{0}(\rho)}=\left(\frac{\pi}{4}\right)^{2}\left(\frac{\Theta}{\lambda / D}\right)^{2}$,

which means that the effect of a nuller coronagraph is decoupled from the effect of apodization. This expression also shows that the addition of a nuller coronagraph to an apodized coronagraph is relevant when the apparent star diameter $\Theta$ is small enough with respect to the resolution unit $\lambda / D$.

A few illustrative examples of the star leakage are given in Figs. 5 to 8 . To draw these curves, it is necessary to take fixed values for the parameters $\varepsilon$ (the flux ratio planet to star) and $\Theta$. In the following figures, the underlying astronomical target is a solar-like system at $10 \mathrm{pcs}$, the stellar diameter is then 1 mas (milli arc second). In Fig. 5 we compared the leakage for a $s$ value of 6 to what could be obtained with a clear aperture. These curves are also compared to the PSF for an experiment operated

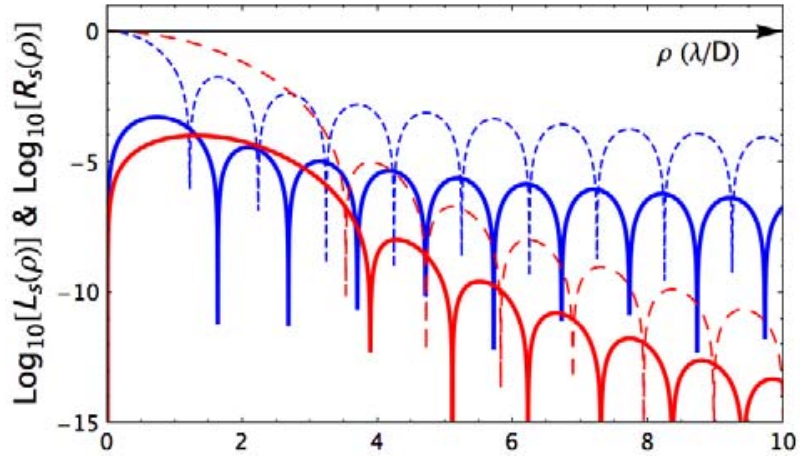

Fig. 5. Continuous curves: star leakage $L_{s}(\rho)$ (logarithmic scale) with $\Theta=0.08 \lambda / D$ for two Sonine apodizations. Top: $s=0$ (clear aperture), bottom: $s=6$. Dashed curves: apodized PSFs $R_{s}(\rho)$ for the same apodizations.

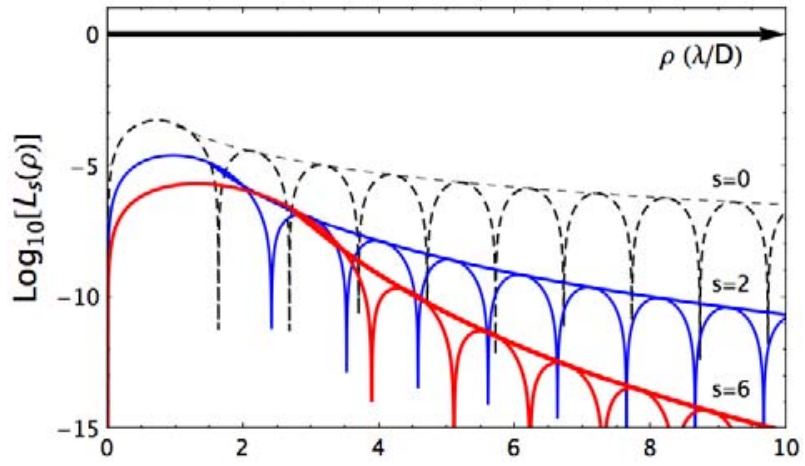

Fig. 6. Star leakage $L_{s}(\rho)$ and the corresponding envelope (logarithmic scale) with $\Theta=0.08 \lambda / D$ for three Sonine apodizations. From top to bottom: $s=0$ (clear aperture, dashed line), 2, 6 (continuous lines). The angular distance $\rho$ is in units of $\lambda / D$. The envelopes start at $1,1.5$, and $2.5 \lambda / D$.

without a NC. The $x$-axis is in units of resolution, the star diameter is of $0.08 \lambda / D$. In Fig. 6 we make a comparison of star leakages for $s$ values equal to 0,2 , and 6 together with the corresponding envelopes in the diffraction wings.

Although so far we have given the expressions of the functions as radial functions, the image formed at the telescope focus must be described in two dimensions. If we denote as $\rho$ the angular vector position in the focal plane, with $|\rho|=\rho$, and $\rho_{\mathrm{P}}$ the angular vector position of the planet, neglecting the possible interferences between the twin images of the planet, the observed intensity in the focal plane is written as

$I_{s}(\rho)=L_{s}(\rho)+\frac{\varepsilon}{4} R_{s}\left(\left|\rho-\rho_{\mathrm{P}}\right|\right)+\frac{\varepsilon}{4} R_{s}\left(\left|\rho+\rho_{\mathrm{P}}\right|\right)$.

The intensity is reduced by a factor of 4 because of the partition of the planet light into four images (two-twin images at each of the two outputs of the system). Figure 7 shows what could be obtained for an Earth-like planet (at 100 mas of the star), with $\varepsilon=10^{-10}$. The observation is made with a $10-\mathrm{m}$ telescope at $600 \mathrm{~nm}$. A comparison is made in the figure of what could be obtained with an AIC alone (unapodized), with apodization alone and when coupling apodization with an AIC. For the representation of the apodization alone image, the background used is the mere PSF, e.g. ignoring the smoothing effect of the star (the convolution between the PSF and the star image). In fact, numerical simulations that are not reported here for the sake of conciseness show that the effect is insubstantial as long as the star diameter remains smaller than $\lambda / D$. 

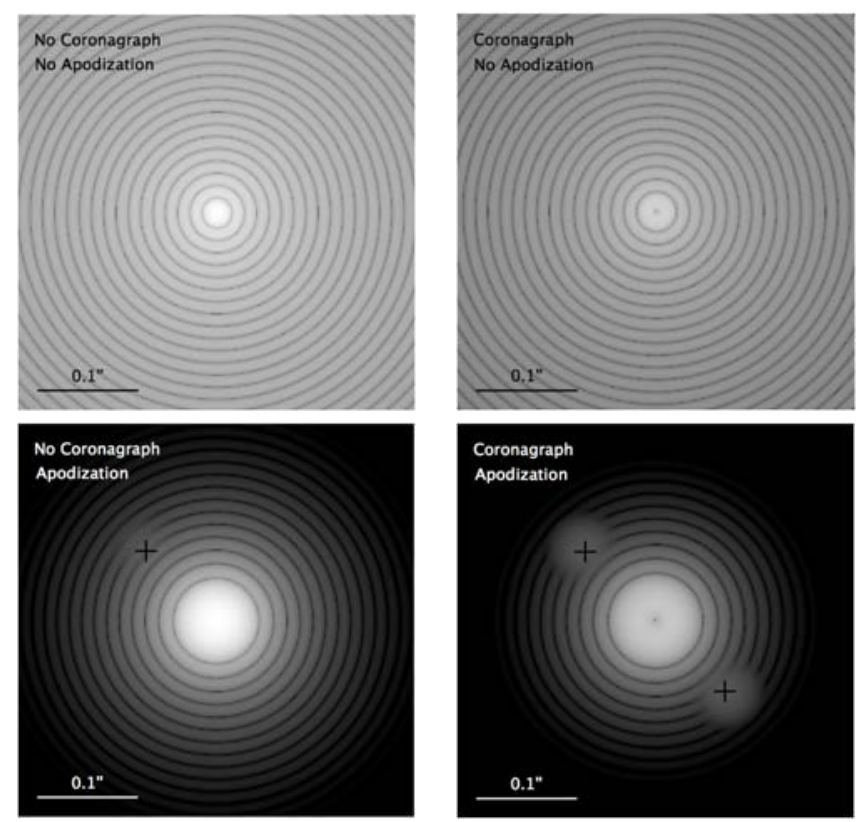

Fig. 7. Intensity in logarithmic scale of a $10 \mathrm{pc}$ distant star and a planet located at $1 \mathrm{AU}$ from it $\left(0.1^{\prime \prime}\right)$ with a flux ratio of $10^{10}$ for a $10 \mathrm{~m}$ aperture. Left: without a nuller coronagraph. Right: with a nuller coronagraph. Top: with a clear aperture. Bottom: with a Sonine-apodized aperture $s=6$. The intensity scale is the same as in Fig. 8 and goes from -15 (black) to 0 (white). The crosses in the two bottom images indicate the position of the planet.
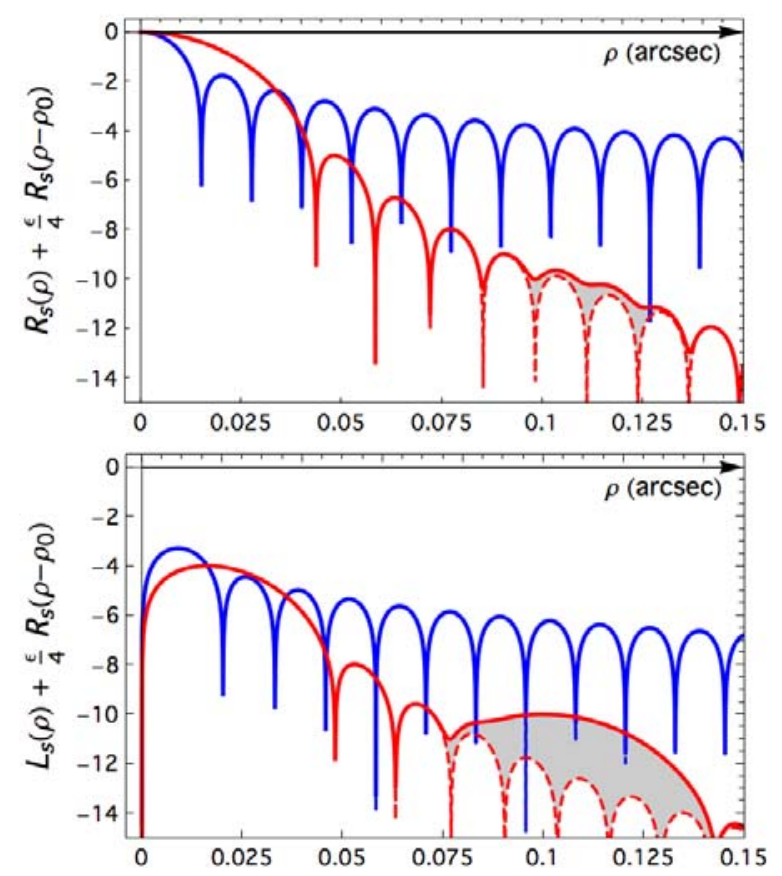

Fig. 8. Intensity in logarithmic scale of a $10 \mathrm{pc}$ distant star and a planet located at $1 \mathrm{AU}$ from it $\left(0.1^{\prime \prime}\right)$ with a flux ratio of $10^{10}$. The diameter of the telescope is $10 \mathrm{~m}$ and the observation wavelength $600 \mathrm{~nm}$. Top: without a nuller coronagraph, bottom: with a nuller coronagraph. The cases of a clear aperture (blue curve) and apodized aperture (red curve) are compared. The dashed curves correspond to the image of the star alone. The gray areas highlight the contributions of the planet.

The figure shows that the visibility of the Earth-like planet is significantly improved by coupling an apodizer with a NC, and reciprocally a system based on apodization alone will highly benefit from the introduction of a NC. Of course the gain will

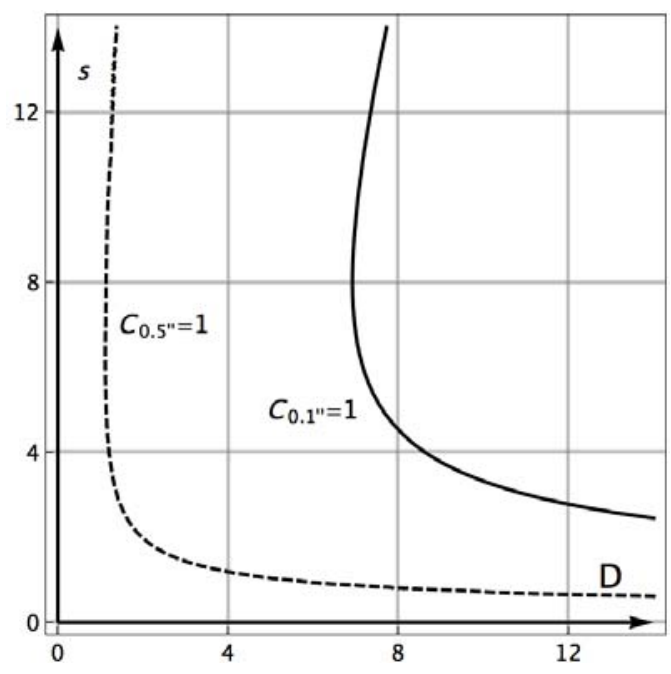

Fig. 9. Set of points in the $D \times s$ plane such that $C_{s}\left(\rho_{\mathrm{P}}\right)=1$ for a planet at $0.1^{\prime \prime}$ (continuous curve) and at $0.5^{\prime \prime}$ (dashed curve) from the star (apodization parameter $s$ and telescope diameter $D$ ). The intensity ratio star/planet is $10^{10}$. The curves show that there is a minimal diameter size required for exoplanet detection.

strongly depend on the value of $\Theta$, as shown by Eq. (24). Cuts of these images are shown in Fig. 8 for a Sonine-apodized aperture of $s=6$ with and without a NC. While the planet is located at 100 mas from the star, its diffraction spot may appear to be centered at a different location. This observational bias is caused by the logarithmic scale used to represent the data, and also because the star leakage is not constant over the area of the planet's image.

\section{Contrast and signal-to-noise ratio considerations}

We consider in this section two quantities, the local contrast and the signal-to-noise ratio $(S N R)$, which might appear similar but are essentially different because the contrast is an estimator that assumes a classical detection of the light while the SNR takes into account the photodetection process leading to the socalled photon noise. Both quantities are useful but the $S N R$ is the ultimate quantity that can define whether a detection can be achieved for a given wavelength, bandwidth, etc. The contrast remains an interesting quantity however because it will be difficult in practice if the contrast is not high enough to distinguish between the planet and unwanted perturbative effects such as the residual speckles not considered in this analysis.

We define the local contrast as the ratio between the height $\frac{\varepsilon}{4} R_{s}(0)$ of the twin images of the planet to the level $L_{s}\left(\rho_{\mathrm{p}}\right)$ of the star leakage at the central location $\rho=\rho_{\mathrm{p}}$ of the planet. Using the simplified expression for the envelope of the leakage, we have

$$
\begin{aligned}
& C_{s}\left(\rho_{\mathrm{p}}\right)=\frac{\varepsilon}{4} \frac{R_{s}(0)}{L_{s}\left(\rho_{\mathrm{p}}\right)}=\frac{C_{0}\left(\rho_{\mathrm{p}}\right)}{(1+s)^{2} g_{s}\left(\rho_{\mathrm{p}}\right)}, \text { with } \\
& C_{0}\left(\rho_{\mathrm{p}}\right)=\frac{\varepsilon}{4} \frac{R_{0}(0)}{L_{0}\left(\rho_{\mathrm{p}}\right)}=\frac{\varepsilon}{2} \frac{\pi^{2} D \rho_{\mathrm{p}}^{3}}{\lambda \Theta^{2}} .
\end{aligned}
$$

An illustration of the use of these relations is given in Fig. 9, to derive the optimal diameters and Sonine apodizations for the extra solar system at $10 \mathrm{pc}$ already considered. The curves give the solution of the equation that equals the local contrast to 1 for an exo-Earth and an exo-Jupiter. The value of $\varepsilon$ is fixed to $10^{-10}$ for both exoplanets though, as expected, the exo-Earth is a more difficult target than the exo-Jupiter. We just consider in our illustration the difference of angular separation. In both cases there 
is an optimal value of $s$ that minimizes the necessary aperture diameter. This value is not extremely critical, but if the choice of $s$ is not optimal, the minimum telescope diameter increases substantially. For example, when observing a planet at 0.1 arc$\mathrm{sec}$ from its star, the minimum telescope diameter is $7 \mathrm{~m}$ for the optimal value of $s=8$. For $s=4$, the minimum diameter becomes $8.5 \mathrm{~m}$, and $11 \mathrm{~m}$ for $s=3$. For Jupiter-like planets the minimum diameters correspond to telescopes down to the $2 \mathrm{~m}$ class.

We define the $S N R$ as the ratio between the number of photons $N_{\mathrm{P}}$ gathered from the planet's twin images and the statistical fluctuations produced by the star leakage. Denoting with $N_{\mathrm{L}}$ the number of photons of the star leakage underneath the planet images, the total number of photons at the planet position is $N_{\mathrm{L}}+N_{\mathrm{P}}$. Assuming a Poisson process for the photodetection, the root mean square of the statistical fluctuations is $\sqrt{N_{\mathrm{L}}+N_{\mathrm{P}}}$, which reduces to $\sqrt{N_{\mathrm{L}}}$ for a low contrast. The expression of the SNR is then

$S N R=\frac{N_{\mathrm{P}}}{\sqrt{N_{\mathrm{L}}+N_{\mathrm{P}}}} \cdot$

The numbers $N_{\mathrm{P}}$ and $N_{\mathrm{L}}$ can be written as

$$
\begin{aligned}
& N_{\mathrm{P}}=k \frac{\varepsilon}{2} \Phi_{s}=k \frac{\varepsilon}{2(1+2 s)} \Phi_{\mathrm{O}} \\
& N_{\mathrm{L}}=2 k \triangle_{s} L_{s}\left(\rho_{\mathrm{p}}\right)=2 k \triangle_{0} L_{0}\left(\rho_{\mathrm{p}}\right) \frac{(1+s)^{2}}{1+2 s} g_{s}\left(\rho_{\mathrm{p}}\right),
\end{aligned}
$$

where $k$ is the factor that converts the energy collected by the experiment into a number of photons per unit of surface.

It can be evaluated through the standard expression:

$k=\eta \frac{F_{\text {ref }}}{h \nu} 10^{-0.4 m} \Delta \lambda \tau$,

where $\eta$ stands for the efficiency of the optical system including the quantum efficiency of the detector, $F_{\text {ref }}$ is the reference flux, $m$ is the apparent magnitude of the star, $\Delta \lambda$ is the bandwidth, and $\tau$ is the exposure time.

We chose to consider a bandwidth of $88 \mathrm{~nm}$ centered at $\lambda=555 \mathrm{~nm}$, which makes it very similar to the $V$ band. The value of $\eta$ was chosen to be 0.5 . The solar-like star being at $10 \mathrm{pc}$, its apparent magnitude is therefore the same as its absolute magnitude $(m=4.83)$. In these conditions the value of $k$ is $4.91 \times 10^{7}$ photons per surface unit for an exposure time of $1 \mathrm{~s}$.

Figure 10 is a representation similar to Fig. 9, but considering the $S N R$ instead of contrast. It displays the evolution of the telescope's diameter as a function of $s$ for three different values of the $S N R(1,5$, and 10) obtained with an exposure time of $1 \mathrm{~h}$. For low values of the $S N R$ the curves are similar to those of the contrast but higher values of the $S N R$ lead to higher curvatures. For $S N R$ s of 5 and 10 and for both planets these functions have the same asymptotes. As for the contrast, minimum values of $D$ are found for specific $s$.

Figure 11 represents the optimal $s$ as a function of $D$ for the Earth-like and the Jupiter-like planets. A maximum $s_{\max }$ is reached for a particular value of $D$. The closer to the star, the higher $s_{\max }$, because the star leakage, which is also higher, has to be compensated for. That we associated an optimal apodization to a given diameter $D$ does not mean that this pairing will be efficient for any astronomical target. One may wonder if an apodizer based on the same optimal $s$ can be used to observe two planets at different angular separations $\rho_{1}$ and $\rho_{2}$ from their star. It is true that a unique, common pair $(D, s)$ can be found at the intersection of the two curves $D_{\rho_{1}}(s)$ and $D_{\rho_{2}}(s)$. However, if we

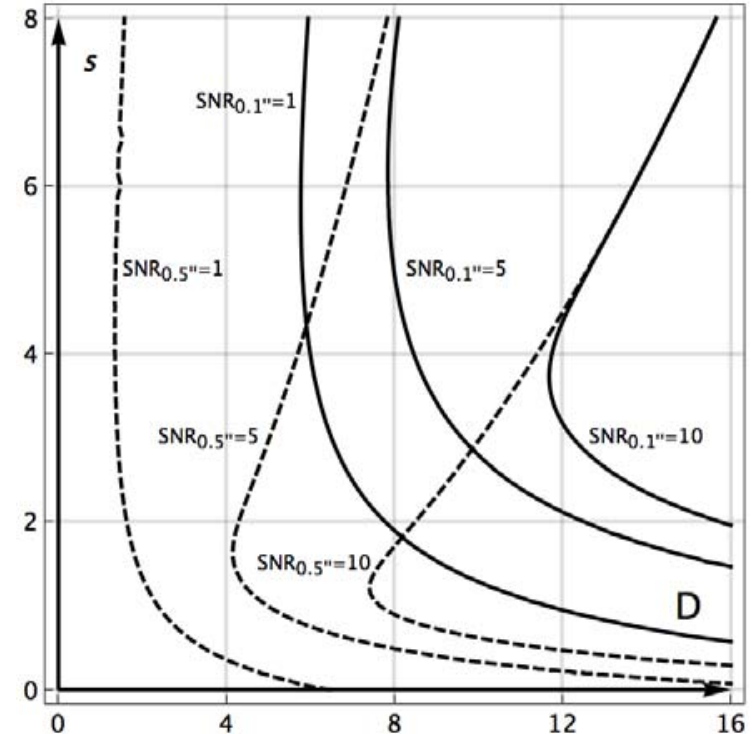

Fig. 10. Set of points in the $D \times s$ plane such that $S N R_{s}\left(\rho_{\mathrm{P}}\right)=1,5$, and 10 , for angular distances $\rho_{\mathrm{P}}=0.5^{\prime \prime}$ (dashed curves) and $0.1^{\prime \prime}$ (continuous curves). The intensity ratio star/planet is $10^{10}$, the other observing parameters are given in the body of the paper. There is a minimal diameter size required to reach a given $S N R$ value.

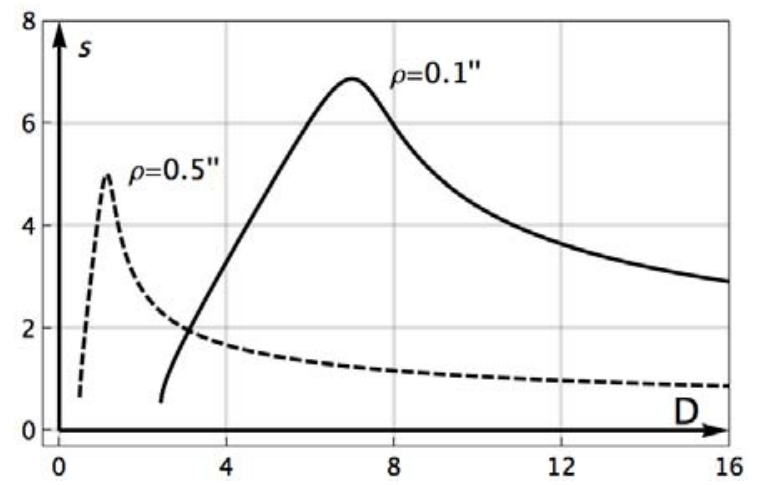

Fig. 11. Optimal Sonine parameter $s$ as a function of the telescope diameter $D$ for the Earth-like planet (continuous curve) and the Jupiter-like planet (dashed curve).

consider the case highlighted in Fig. 11 , we have $D \approx 3 \mathrm{~m}$ and $s \approx 2$. If the $S N R$ associated with the detection of the Jupiter-like planet can reach 10 for an exposure time of almost $9 \mathrm{~h}$, the same SNR cannot be reached when looking for the Earth-like planet unless the exposure time exceeds 8.7 years.

A telescope of diameter $8 \mathrm{~m}$ observing for one hour offers the possibility of detecting planets at 0.1 arcsec and 0.5 arcsec for $S N R$ s of respectively 5 and 10 (but for different values of $s)$. Table 1 gives an extensive list of the exposure times needed to detect planets at these two angular distances for telescope diameters of 2,10, and $30 \mathrm{~m}$. Although a $2 \mathrm{~m}$ telescope cannot detect a planet at 0.1 arcsec in a reasonable amount of time (the limit was set to $10^{5} \mathrm{~s}$, which is a little more than one day), a $10 \mathrm{~m}$ telescope can achieve this detection with an SNR of 10 for an exposure time of $1.6 \mathrm{~h}$. Even if the star-leakage effect is higher for bigger telescope, this time decreases to less than 6 min for a $30 \mathrm{~m}$ telescope. The apodization parameters associated with these detections are smaller than those found by looking at Fig. 9.

\section{Discussion and conclusion}

We showed that using an apodized aperture with a nuller coronagraph leads to a decisive improvement of the detection 
Table 1. Required exposure times and optimal apodization parameters to detect the Earth-like and Jupiter-like planets for three different telescopes' diameters with three different SNRs.

\begin{tabular}{|c|c|c|c|c|c|c|c|}
\hline \multirow[b]{2}{*}{$\rho$} & \multirow[b]{2}{*}{ SNR } & \multicolumn{2}{|c|}{$D=2 \mathrm{~m}$} & \multicolumn{2}{|c|}{$\bar{D}=10 \mathrm{~m}$} & \multicolumn{2}{|c|}{$\bar{D}=30 \mathrm{~m}$} \\
\hline & & $t$ & $s$ & $t$ & $s$ & $t$ & $s$ \\
\hline & 10 & & & $5750 \mathrm{~s}$ & & $315 \mathrm{~s}$ & \\
\hline $0.1^{\prime \prime}$ & $\begin{array}{l}5 \\
1\end{array}$ & N.A. & N.A. & $\begin{array}{c}1425 \mathrm{~s} \\
58 \mathrm{~s}\end{array}$ & 4.4 & $\begin{array}{c}78 \mathrm{~s} \\
3 \mathrm{~s}\end{array}$ & 2 \\
\hline & 10 & $96000 \mathrm{~s}$ & & $1800 \mathrm{~s}$ & & $150 \mathrm{~s}$ & \\
\hline $0.5^{\prime \prime}$ & $\begin{array}{l}5 \\
1\end{array}$ & $\begin{array}{c}24000 \mathrm{~s} \\
960 \mathrm{~s}\end{array}$ & 2.7 & $\begin{array}{c}455 \mathrm{~s} \\
18 \mathrm{~s}\end{array}$ & 1.1 & $\begin{array}{l}38 \mathrm{~s} \\
1.5 \mathrm{~s}\end{array}$ & 0.7 \\
\hline
\end{tabular}

performance. The apodization has two major consequences: the first is that it allows the coronagraph to greatly reduce the onsky transmission of slightly off-axis sources of light. The starleakage is thus reduced, as are small pointing errors. The second is that, as can be observed in other apodized coronagraphs, the apodization also helps concentrating the diffracted energy in the central lobe, which improves the detectability of the companion.

Given an angular separation between the star and the planet, there is a minimum telescope diameter (associated with a specific apodization parameter $s$ of the Sonine function) that allows a detection with a sufficient local contrast ratio, or a sufficient $S N R$ for a typical exposure time. In the specific case of the Sonine functions, detecting an exo-earth around a solar-type star at $10 \mathrm{pc}$ with an $S N R$ of 10 can be done in $1.6 \mathrm{~h}$ with a $10 \mathrm{~m}$ telescope and an apodization parameter $s$ of 4.4 , or in less than $6 \mathrm{~min}$ if the telescope has a diameter of $30 \mathrm{~m}$ (and the apodization strength is in this case 2). The study proposed here has been solely limited to Sonine apodizers because they make it possible to obtain algebraic expressions for the star leakage. Other apodization profiles might be more efficient than Sonine apodizers however, and may be used in practice, even if it will not be possible to express their effect using analytic expressions. Some candidates among the possible apodization profiles are the spheroidal prolate functions (Slepian 1965) that, as was well described in Frieden (1971), maximally concentrate in the mean square sense the light in the diffraction central spot. The properties of the spheroidal prolate functions make them very interesting for the Lyot coronagraph, as was pointed out in several papers (Aime et al. 2002; Soummer et al. 2003; Soummer 2005).

The coupling of a nuller coronagraph and an apodizer has an additional advantage: it relaxes their respective specifications, in particular for apodizers with a continuously variable transmission. Nevertheless, the difficulty of manufacturing these apodizers may lead the observer to use microdot apodizers, as studied in Martinez et al. (2010) in the context of the prolate-apodized Lyot coronagraph (PALC) of the ESO-Sphere project (and possibly for the coronagraphic instrument of the E-ELT). Micron-size dots with a transmission of zero are deposited on a substrate. This type of apodizer is therefore a binary apodizer. The density of the dots is such that it approximates the apodization profile. This type of apodizer has also been selected and tested for the PALC of the Gemini Planet Imager project (Sivaramakrishnan et al. 2010). As noted by the authors, the design of microdot apodizers has to be carefully chosen because the diffraction from the size of the microdots can create unwanted effects on the PSF.

There are several other types of binary masks. Some early examples have been described in Boivin (1952) and Jacquinot \& Roizen-Dossier (1964). They were more recently studied in detail for direct detection of exoplanets. Besides, numeric optimizations of coronagraphic problems can lead to various designs of pupil masks: shaped pupils, barcode masks, checkerboard masks, and other types of binary apodizers are described in Spergel \& Kasdin (2001), Vanderbei et al. (2003), and Kasdin et al. (2003). These apodizers can provide very high contrasts and possess the advantage of having achromatic transmissions. The Space Infrared telescope for Cosmology and Astrophysics (SPICA, Spica Working Group et al. 2010) for example has selected checkerboard masks (among other types of pupil masks) for its coronagraph.

Finally, apodization can also be obtained using a phase induced amplitude apodizer (PIAA, see Guyon 2003; Vanderbei 2006). The specific properties of PIAA are complex however and cannot be introduced in passing in the present analysis. Whatever the apodizing technique is, combining it with a nuller coronagraph can greatly improve the detection capabilities of the instrument.

\section{References}

Aime, C. 2005, A\&A, 434, 785

Aime, C. 2007, C. R. Phys., 8, 273

Aime, C., Soummer, R., \& Ferrari, A. 2002, A\&A, 389, 334

Aime, C., Ricort, G., Carlotti, A., Rabbia, Y., \& Gay, J. 2010, A\&A, 517, A55

Allouche, F., Glindemann, A., Aristidi, E., \& Vakili, F. 2009, A\&A, 500, 1277

Arenberg, J. W., Lo, A. S., Glassman, T. M., \& Cash, W. 2007, C. R. Phys., 8, 438

Baudoz, P., Rabbia, Y., \& Gay, J. 2000a, A\&AS, 141, 319

Baudoz, P., Rabbia, Y., Gay, J., et al. 2000b, A\&AS, 145, 341

Boivin, A. 1952, J. Opt. Soc. Am. (1917-1983), 42, 60

Bracewell, R. N. 1978, Nature, 274, 780

Cash, W. 2006, Nature, 442, 51

Frieden, B. R. 1971, Progress in Optics, ed. E. Wolf (North-Holland Publishing, Co.)

Gay, J., \& Rabbia, Y. 1996, Academie des Science Paris Comptes Rendus Ser. B Sci. Phys., 322, 265

Guyon, O. 2003, A\&A, 404, 379

Guyon, O. 2009, in Am. Inst. Phys. Conf. Ser., ed. T. Usuda, M. Tamura, \& M. Ishii, 1158,285

Guyon, O., Pluzhnik, E. A., Kuchner, M. J., Collins, B., \& Ridgway, S. T. 2006, ApJS, 167, 81

Jacquinot, P., \& Roizen-Dossier, B. 1964, Progress in Optics, ed. E. Wolf (NorthHolland Publishing, Co.), 31

Kasdin, N. J., Vanderbei, R. J., Spergel, D. N., \& Littman, M. G. 2003, ApJ, 582, 1147

Martinez, P., Dorrer, C., Kasper, M., Boccaletti, A., \& Dohlen, K. 2010, A\&A, 520, A 110

Mawet, D., Riaud, P., Absil, O., Baudrand, J., \& Surdej, J. 2005, in SPIE Conf., ed. D. R. Coulter, 5905, 502

Nisenson, P., \& Papaliolios, C. 2001, ApJ, 548, L201

Nishikawa, J., Kotani, T., Murakami, N., et al. 2005, A\&A, 435, 379

Oppenheimer, B. R., \& Hinkley, S. 2009, ARA\&A, 47, 253

Osterberg, H., \& Wilkins, Jr., J. E. 1949, J. Opt. Soc. Am. (1917-1983), 39, 553

Perryman, M. A. C. 2000, Reports Progr. Phys., 63, 1209

Rabbia, Y., Baudoz, P., \& Gay, J. 1998, in The Next Generation Space Telescope: Science Drivers and Technological Challenges, ed. B. Kaldeich, ESA Special Publication, 429, LIA Colloq., 34, 279

Roddier, F., \& Roddier, C. 1997, PASP, 109, 815

Rouan, D., Riaud, P., Boccaletti, A., Clénet, Y., \& Labeyrie, A. 2000, PASP, 112, 1479

Seager, S., \& Sasselov, D. D. 2000, ApJ, 537, 916

Sivaramakrishnan, A., Soummer, R., Oppenheimer, B. R., et al. 2010, in SPIE Conf., 7735

Slepian, D. 1965, J. Opt. Soc. Am. (1917-1983), 55, 1110

Soummer, R. 2005, ApJ, 618, L161

Soummer, R., Aime, C., \& Falloon, P. E. 2003, A\&A, 397, 1161

Spergel, D., \& Kasdin, J. 2001, in American Astronomical Society Meeting Abstracts, BAAS, 33, 1431

Spica Working Group, Enya, K., \& SPICA Working Group. 2010, Adv. Space Res., 45, 979

Tavrov, A. V., Otani, Y., Kurokawa, T., \& Takeda, M. 2005, in SPIE Conf. Ser., 5905, ed. D. R. Coulter, 398

Tavrov, A. V., Nishikawa, J., Tamura, M., et al. 2008, Appl. Opt., 47, 4915

Vanderbei, R. J. 2006, ApJ, 636, 528

Vanderbei, R. J., Spergel, D. N., \& Kasdin, N. J. 2003, ApJ, 599, 686

Vanderbei, R. J., Cady, E., \& Kasdin, N. J. 2007, ApJ, 665, 794

Wright, J. T., Fakhouri, O., Marcy, G. W., et al. 2011, PASP, accepted [arXiv: 1012 . 5676] 